\title{
On the coupling influence of the relative position of the human trunk with respect to overhead high-voltage power lines
}

\author{
Marius Valerian Paulet ${ }^{1}$, Alexandru Salceanu ${ }^{1}$, Constantin Dan Neagu ${ }^{1}$, Dragos Florin Bordeianu ${ }^{1}$ \\ 1 'Gheorghe Asachi' Technical University of Iasi, Romania, Faculty of Electrical Engineering
}

\begin{abstract}
In this article, we present our study of the relevance of the relative position of the human torso with respect to the plane of the supporting pylons of various Overhead High-Voltage Power Lines from the perspective of currents induced by the generated magnetic fields. A homogenous model of the human body that considers the trunk as an elliptic cylinder has been developed in CST Studio software. Due to the necessary theoretical brevity, the results obtained by the simulation for the loop currents along the perimeters associated with the large $(28 \mathrm{~cm})$ and, respectively, the small $(16 \mathrm{~cm})$ axis of the elliptical cross-section of the trunk are presented and discussed. Simulations have been performed for five relative positions $\left(0^{\circ}, 30^{\circ}, 45^{\circ}, 60^{\circ}, 90^{\circ}\right)$ of the human trunk with respect to the plane of the transmission towers and for two types of symmetric, double three-phase networks ( $110 \mathrm{kV}$ and $640 \mathrm{~A}, 220 \mathrm{kV}$ and $960 \mathrm{~A}$, respectively), with the normal supporting poles SN 110252. We present the solutions and the selected boundary conditions with the aim of using CST software in the domain of industrial frequency. The obtained results make it possible to formulate recommendations on the reduction of human exposure to magnetic fields.
\end{abstract}

\section{Section: RESEARCH PAPER}

Keywords: magnetic fields; high-voltage power lines; Induced currents; CST simulation

Citation: Marius Valerian Paulet, Alexandru Salceanu, Constantin Dan Neagu, Dragos Florin Bordeianu, On the coupling influence of the relative position of human trunk with respect to the overhead high-voltage power line, Acta IMEKO, vol. 9, no. 3, article 9, September 2020, identifier: IMEKO-ACTA-09 (2020)03-09

Editor: Jan Saliga, Technical University of Košice, Slovakia

Received February 20, 2020; In final form March 23, 2020; Published September 2020

Copyright: This is an open-access article distributed under the terms of the Creative Commons Attribution 3.0 License, which permits unrestricted use, distribution, and reproduction in any medium, provided the original author and source are credited.

Corresponding author: Alexandru Salceanu, e-mail: asalcean@tuiasi.ro

\section{MAGNETIC AND ELECTRIC COUPLING BETWEEN OVERHEAD HIGH-VOLTAGE POWER LINES AND THE HUMAN BODY}

High-voltage (and also high current) power transmission networks are sources of considerable magnetic and electric fields. Specifically, these are voltages of hundreds of $\mathrm{kV}$, generating significant electric fields and currents above $1000 \mathrm{~A}$, which produce magnetic fields that should be taken into account. Most of the relevant concerns relate to the influence that these fields could have on living matter.

Shielding (and, implicitly, protection) from electric fields is easier to achieve than from magnetic ones. Any „shell” (be it very thin or with numerous apertures) with conductive properties can play the protective role of a Faraday cage.

Matters are more complicated in the case of magnetic fields, which are associated with moving electrical charges. The techniques for reducing the possible inductive coupling are few and expensive. Those that do not impose unacceptable costs or dimensions are primarily based on increasing the distance from the source.

In Romania, the National Power Transmission Network, operated by the National Company 'Transelectrica', measures approximately $9000 \mathrm{~km}$ of overhead power lines (the vast majority being $220 \mathrm{kV}$ and $400 \mathrm{kV}$ lines) and 81 power stations (approximately the same parity, 42 of $220 \mathrm{kV}, 38$ of $400 \mathrm{kV}$, and only one of $750 \mathrm{kV}$ ). In addition, thousands of kilometres of $110 \mathrm{kV}$ overhead power lines (managed by various distribution companies) should be also considered. Of course, $110 \mathrm{kV}$ represents the theoretical, conventional 'boundary' between medium and high voltage, but due to the lower height of the supporting pylons and the greater proximity to the inhabited areas, the $110 \mathrm{kV}$ lines must be also included due to their possible harmful effects on health in the same category as $220 \mathrm{kV}, 400 \mathrm{kV}$, or even $750 \mathrm{kV}$ lines. 
Values that are not considered dangerous and therefore acceptable, for current densities induced in the human body by electric fields with frequencies lower than $100 \mathrm{kHz}$ (capacitive coupling) or by magnetic fields with the same frequency spectrum (inductive coupling) are known in the literature under the generic name of 'basic restrictions'. These values have mainly been established by considering the instant, short-term health effects: the excitation of nerves and muscles.

The human body is electrically non-homogeneous; therefore, the values of the basic restrictions are expressed in the form of the current density obtained by averaging the current over a $1 \mathrm{~cm}^{2}$ cross-section, perpendicular to the current flow.

A complicated and debatable issue is setting the correspondence between the electric and magnetic fields that exist in the surrounding environment (the so-called reference levels) and the current density actually induced in the human body.

Numerous models based on the human anatomy (often based on Magnetic Resonance Imaging, MRI) have been developed with millimetre resolutions. Using these models as well as numerical methods of solving (partial) differential equations (the finite element method or finite difference method), the currents induced in the human body model by the electric and magnetic fields in the immediate vicinity of the human subject can be calculated.

We must then compare the value of the current density induced in various organs with the typical limits accepted by the standards [1]: $10 \mathrm{~mA} / \mathrm{m}^{2}$ (professional, occupational exposure) and only $2 \mathrm{~mA} / \mathrm{m}^{2}$ (residential, public exposure).

Since coupling mechanisms are fundamentally different, the vast majority of simulation/modelling programs have different modules for low and, respectively, high frequencies. Moreover, at a low frequency, there are two different solvers: one is (quasi)electrostatic and is recommended when the dominant field is the electric one, and the other is (quasi)magnetostatic and suitable for the dominant magnetic field occurrence.

Here, we must specify the criterion that allows us to determine the nature of the dominant field because due to the different units of measure, $\mathrm{V} / \mathrm{m}$ and $\mathrm{A} / \mathrm{m}$, it is not possible to compare them directly with each other. What could be compared are the associated volumetric energy densities, expressed in $\mathrm{J} / \mathrm{m}^{3}$ :

$$
W_{B}=\frac{B \cdot H}{2}=\frac{1}{2} \mu_{0} H^{2} \text { and } W_{E}=\frac{D \cdot E}{2}=\frac{1}{2} \varepsilon_{0} E^{2} .
$$

In the case of high-voltage power lines, the problem of the nature of the dominant field is of particular importance. We have both very high voltages and very high currents. In the case of the equivalence of the spatial energy densities of the two fields, we obtain the well-known value of the wave impedance [2] as the ratio between the electric field strength and the magnetic field strength (in free space):

$$
\begin{aligned}
\frac{1}{2} \mu_{0} H^{2}=\frac{1}{2} \varepsilon_{0} E^{2} & \Rightarrow \frac{E}{H}=\sqrt{\frac{\mu_{0}}{\varepsilon_{0}}}=\sqrt{\frac{4 \pi \cdot 10^{-7}}{\frac{1000}{36 \pi} 10^{-12}} \Omega} \\
& =120 \pi \Omega \simeq 377 \Omega .
\end{aligned}
$$

If at the point of interest, the $E / H$ ratio is greater than $377 \Omega$, the dominant field is the electric one, but if this ratio is less than $377 \Omega$, the dominant field becomes the magnetic one. The electric field strength is proportional to the scalar electrical potential and is higher if electric charges are nearer to the measuring point.
Some approximations, which are very close to reality, should be made, especially for $50 / 60 \mathrm{~Hz}$ frequencies (the so-called mains frequency), which fall under the extremely low frequency category.

Most important for the (quasi)electrostatic approach is neglecting the time respect variations of the electric flux density in the Ampere law (written in harmonic mode).

In this way, the influence of the electric field on the current density in conductors in general and in the human body in particular can be properly modelled.

$$
\begin{aligned}
\vec{\nabla} X \vec{H}=\vec{J}+\frac{\partial \vec{D}}{\partial t} & =\sigma \vec{E}+\varepsilon \frac{\partial \vec{E}}{\partial t}=\sigma \vec{E}+j \omega \varepsilon \vec{E} \\
& =(\sigma+j \omega \varepsilon) \vec{E}
\end{aligned}
$$

Around the industrial frequency spectrum, the displacement current in the human body has a much smaller influence than the conduction current.

For the human body at the $50 \mathrm{~Hz}$ frequency, the conductivity $\sigma$ is about $0.2 \mathrm{~S} / \mathrm{m}$, and the relative permittivity $\varepsilon_{\mathrm{r}}$ is approximately $10^{7}$.

An approximate calculation of the product $\omega \varepsilon$ gives the value:

$$
\begin{aligned}
\omega \varepsilon=2 \pi f \varepsilon_{0} \varepsilon_{r}= & 2 \pi \cdot 50 \cdot \frac{1000}{36 \pi} \cdot 10^{-12} \cdot 10^{7}=\frac{1}{36} \\
& \simeq 0.027
\end{aligned}
$$

This value represents about $14 \%$ of the conventionally accepted value for electrical conductivity, which justifies the lower weight of displacement currents. The main weight in the complex conductivity formula belongs to the conduction component rather than to the displacement one. This finding is also valid at frequencies above $50 \mathrm{~Hz}$; regarding the $\omega \varepsilon$ value, the increase in frequency is offset by the decrease in permittivity.

Essentially, the external electric field produces two effects in the human body:

- it establishes a conduction current of the existing charges whose amplitude is determined by the electrical conductivity $\sigma$, and

- it establishes a displacement current, both by forming new charges (bound charges) and by orientating existing dipoles. Permittivity is an expression of the magnitude of polarisation processes in dielectric bodies.

It should be noted that in the case of the human body, both electric conductivity and permittivity vary in a very wide range, depending both on the nature of the tissue and the frequency of the external applied field.

Additionally, an external field due to Coulomb forces will induce a movement of the charges at the surface of the body. This alternate polarisation of superficial charges will cause induced currents, the distribution of which will be determined by both the geometric dimensions of the body and its position relative to the field.

If the magnetic field is dominant (being directly proportional to the current), a magnetostatic solver is used.

The most important approximation for the (quasi)magnetostatic approach is to ignore the magnetic flux density variation in the Faraday-Lenz law, the induced voltage being practically zero:

$$
\vec{\nabla} X \vec{E}=-\frac{\partial \vec{B}}{\partial t} \simeq 0
$$


The interaction between the external variable magnetic field and the human body means the induction of voltages that cause current loops. The larger is the surface intersected by the variable magnetic flux, the higher are the values of the induced voltage. Consequently, the circulating currents have higher values. An indicative formula (deduced from Faraday-Lenz's law) shows that the circular current density $(2 \pi \mathrm{R}$ being the length of the quasi-circular path) is directly proportional not only to the radius of the intersected surface but also to the conductivity of the tissue and to the amplitude and frequency of the magnetic flux density.

$$
J=\pi R f B \sigma
$$

Due to the extensive resources of modern computing systems, most actual simulation/modelling programs of the electromagnetic fields also have a module that takes into account, even at $50 \mathrm{~Hz}$ frequencies, both variations of magnetic flux density and electrical flux density. It should be noted that this option is (computer) resources and time consuming. In the specific case of the CST EM Studio ${ }^{\circledR}$ software [3], this solver is called 'Fullwave', with the name suggesting that both components of the electromagnetic wave are taken into account.

\section{ELLIPTIC CYLINDER MODEL OF THE HUMAN TRUNK SUITED FOR THE STUDY OF INDUCTIVE COUPLING}

The issue of the electric and magnetic fields generated by overhead high-voltage power lines concerns the most diverse teams of researchers, spread across all over the world, using different approaches, but finally reaching convergent conclusions. In the frequency range $50-60 \mathrm{~Hz}$, the biological effects of the electric fields [4] must be treated differently to those of the magnetic fields [5]. A challenging matter is to study the cumulative effects of currents induced by both fields [6]-[8]. Fortunately, the currents induced by the electric field are vertical, and those induced by the magnetic field are in the loop. In addition, there is a phase shift between them, so their maximum values should not be arithmetically summed up. The studies undertaken must be differentiated in terms of the two fundamentally different categories: (1) occupational - for a limited time, a healthy, trained, and protected worker can be found near the high-voltage lines due to some service tasks [9], [10] and (2) residential - when any person, regardless of their age or health status, is located in the vicinity of these lines, which might be placed within newly developed residential districts [11]. Even if $110 \mathrm{kV}$ can be considered the upper limit of medium voltage [12], the generated fields at soil level are comparable with those emitted by higher-voltage networks (as the supporting pylons are lower). One of the problems that called for the attention of researchers is the influence of soil, floor, or footwear on the subject (due to different conductivity and permittivity values) in determining the amplitude of the induced currents in the human body [13].

In [14], we developed an axis-symmetric rotation 3D model consisting of a hemisphere, four cylinders, and four trunks of cone. From the perspective of the current density induced by quasi-homogeneous electric fields along the vertical axis of the body, this model provided values not only in accordance with the analytical calculations but also in accordance with the values in the extant literature.

In [15], we developed a more refined model - closer to the real geometric shapes of the human body. In order to accomplish this target, the trunk has been designed in the shape of an elliptic cylinder (the cross-section is an ellipse with a large radius of $28 \mathrm{~cm}$ and a small one of $16 \mathrm{~cm}$ ). The hands and legs have also been distinctly shaped. This model has proven useful in determining the current density at the body surface and the loop current induced by the horizontal component of the magnetic field generated by overhead high-voltage power lines. The fact that the origin of the three coordinate axes should be considered outside the human body (modelled with two distinct feet) represents a difficulty in determining the vertical induced current.

In the present article, we have aimed to study the influence of the relative position of the human trunk versus the vertical plane determined by the supporting pillars of the network on the magnitude of the induced currents in the body. Emphasis is given to the loop currents induced by the horizontal component of the magnetic field generated by overhead high-voltage power lines. We have developed an intermediate model between the two previously presented ones, also using the CST Suite Studio CAD module in the 'Modelling' section. In particular, we kept the trunk as an elliptic cylinder; however, since the vertical axis of human symmetry should coincide with the vertical axis of the coordinate system, the two legs were modelled together in the form of a truncated cone - which was also elliptic.

\section{THE CST-DEVELOPED MODEL AND THE ACQUIRED RESULTS AND THEIR RELEVANCE}

We have considered five representative positions of the human trunk - the angles between the major axis of the elliptic cross-section and the plane determined by the network pillars: $0^{\circ}, 30^{\circ}, 45^{\circ}, 60^{\circ}$, and $90^{\circ}$ respectively. We have taken into account a double $110 \mathrm{kV}$ three-phase network, with a single grounding conductor, supported by SN 110252 pylons. We have assumed (even for the sake of comparison), two values for the maximum Root Mean Square (RMS) phase current: 640 A (active conductors $2 \times 3$ ACSR 240/40) and 960 A (active conductors $2 \times 3$ ACSR 380/50).

For the model development, we have started from the geometric dimensions of a power transmission network supported by the normal poles SN 110252 (we have chosen the standard case - the normal level to the ground). In Table 1, there are presented the coordinates in the lateral cross-sectional plane; the spatial Cartesian axes have been previously, conveniently fixed.

In this study, we are concerned about the magnetic field generated by overhead high-voltage power lines. For this reason, we did not consider the ground (protective) wire, its contribution being negligible from this perspective.

In [16] and [17], the importance of a correct ground consideration due to the effective contributions of charge and current images was underlined. We have modelled the ground (in a covering way) in the form of a right quadratic prism, with the side of the base being $100 \mathrm{~m}$ and the height (or, more correctly, the depth) being $50 \mathrm{~m}$. We have chosen the soil relative

Table 1. Coordinates $(\mathrm{m})$ of the $2 \times 3$ active wires, the transverse lateral plane, the $-\mathrm{x} 0 \mathrm{x}$ axis being parallel to the wires and normal to this plane.

\begin{tabular}{|c|c|c|c|c|c|c|c|c|c|c|c|}
\hline \multicolumn{2}{|c|}{ R1 } & \multicolumn{2}{|c|}{ S1 } & \multicolumn{2}{|c|}{ T1 } & \multicolumn{2}{|c|}{ R2 } & \multicolumn{2}{|c|}{ S2 } & \multicolumn{2}{|c|}{ T2 } \\
\hline$Y$ & Z & $Y$ & $Z$ & $Y$ & Z & $Y$ & Z & $Y$ & Z & $Y$ & $\mathrm{Z}$ \\
\hline-3.05 & 27.3 & -4.55 & 21.6 & -3.05 & 17 & 3.05 & 27.3 & 4.55 & 21.6 & 3.05 & 17 \\
\hline
\end{tabular}




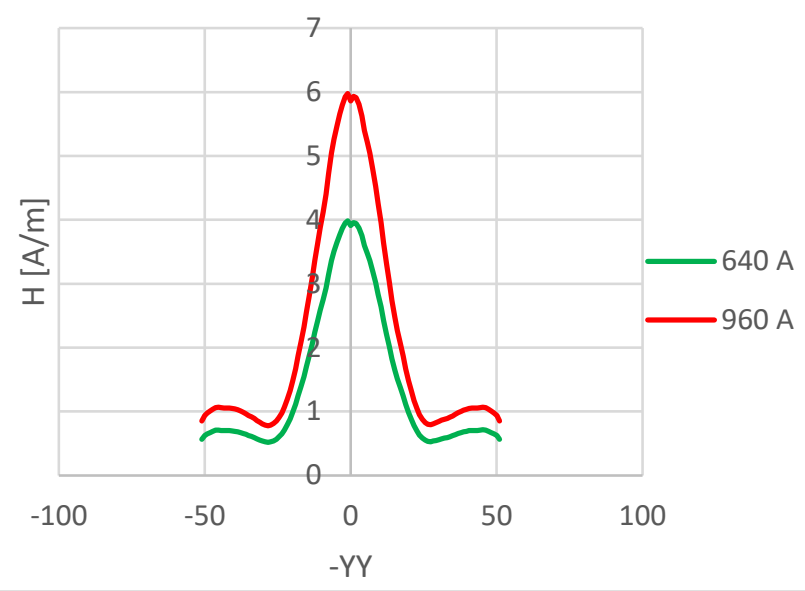

Figure 1. Lateral profile of the magnetic field strength produced at a height of $1 \mathrm{~m}$ from the soil, by two double three-phase $110 \mathrm{kV}$ transmission networks, untransposed. The balanced currents have the RMS values $640 \mathrm{~A}$ and $960 \mathrm{~A}$.

permittivity $\varepsilon_{\mathrm{r}}=40$, while electric conductivity has been fixed at the medium value $10 \mathrm{~S} / \mathrm{m}$. The variation margin for the permittivity of the soil is reduced in comparison with that of the conductivity, which, depending on the nature of the soil and the degree of humidity, can vary from 0.1 to $1000 \mathrm{~S} / \mathrm{m}$.

With the aim of verifying the correctness of the model construction, we have simulated (for two usually encountered RMS values of the current intensity) the lateral profile of the magnetic field generated by the three-phase symmetrical double untransposed network, at the standard height of $1 \mathrm{~m}$ from the soil level. At $1 \mathrm{~m}$ above the ground, most of the vital internal organs (heart, liver, kidneys, stomach) of a man of average height are located.

The result is shown in Figure 1 and agrees well with other simulations presented in the literature [10], [11] and with the individually performed analytical calculations.

The next step was to place the human model in the symmetry centre of the grounding plane (the intersection of the three xyz coordinate axes) in order to set its electrical properties (the relative permittivity $\varepsilon_{\mathrm{r}}$ was $10^{7}$ while the electric conductivity was fixed at the medium value $0.2 \mathrm{~S} / \mathrm{m}$ ) and primarily to establish the

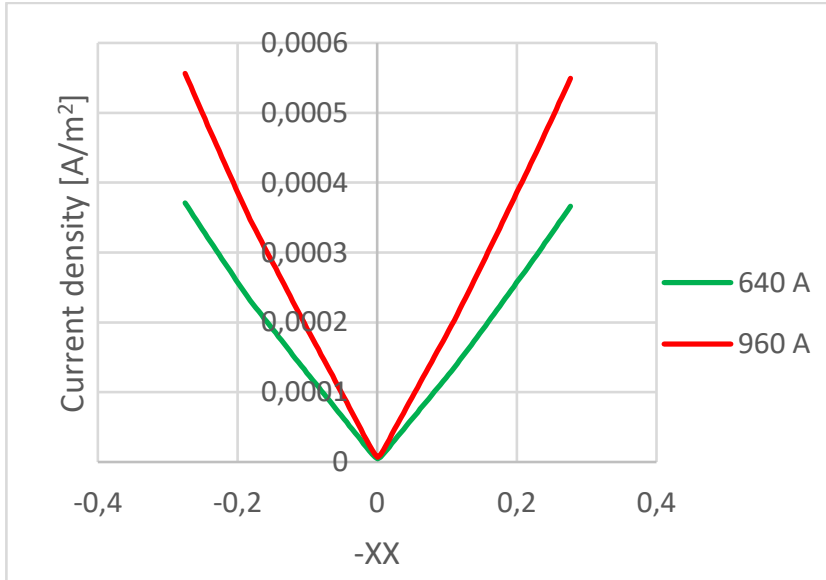

Figure 2. Maximal coupling along the perimeter associated with the large radius, the trunk being parallel to the plane of the pillars. right boundary conditions (a good balance between accuracy and duration).

More exactly, in the magnetostatic solver, on the $-\mathrm{x} 0 \mathrm{x}$ axis boundaries of the electric type were imposed on both ends, while on the $-\mathrm{y} 0 \mathrm{y}$ and $-\mathrm{z} 0 \mathrm{z}$ axes, the 'open-boundary' option was also chosen for both ends.

As for the soil-air working space, we have taken into account two restrictions. Along the $-\mathrm{x} 0 \mathrm{x}$ axis, due to the conductive electric type boundaries, it is compulsory for these extremities to be connected to soil, allowing the current loop to close through the soil. This condition is not for axes $-\mathrm{yOy}$ and $-\mathrm{zO} z$, where a gap between the ground and the open boundary is accepted.

In Figure 2(a), the general defining framework and the previously described human model being placed on the earth in the centre of the selected square surface are presented. The six considered three-phase currents are parallel to the soil.

In [18], we presented compelling simulations and results that demonstrate the irrelevant influence of the trunk orientation upon the surface current density distribution. This conclusion was, in principle, expected in this current study, with the incidental electric field variations being virtually negligible. In Figure 2(b) and (c), there is a progressive colour-coded presentation of these 'scatterings' for the extreme positions of the trunk (parallel and normal to the network plane).

In the same article, we also investigated the vertical currents induced by these fields - they were four orders of magnitude smaller than the accepted basic restrictions, which makes them practically irrelevant.

We have focused on the loop currents induced by the generated magnetic field (see Figure 1) in the trunk of the elliptic cylinder torso model accepted here.

As shown in Equation (6), the induced current density is directly proportional to the radius of the surface perpendicular to the horizontal lines of magnetic field.

In Figure 3, we present the induced current density in the horizontal elliptical cross-section made $1 \mathrm{~m}$ high from the ground. The great axis of the ellipse is parallel to the network plane. Following this assumption, the magnetic inductive coupling reaches its highest value (according to network orientation). It should be noted that for both current intensity RMS values, the induced current density considerably exceeds

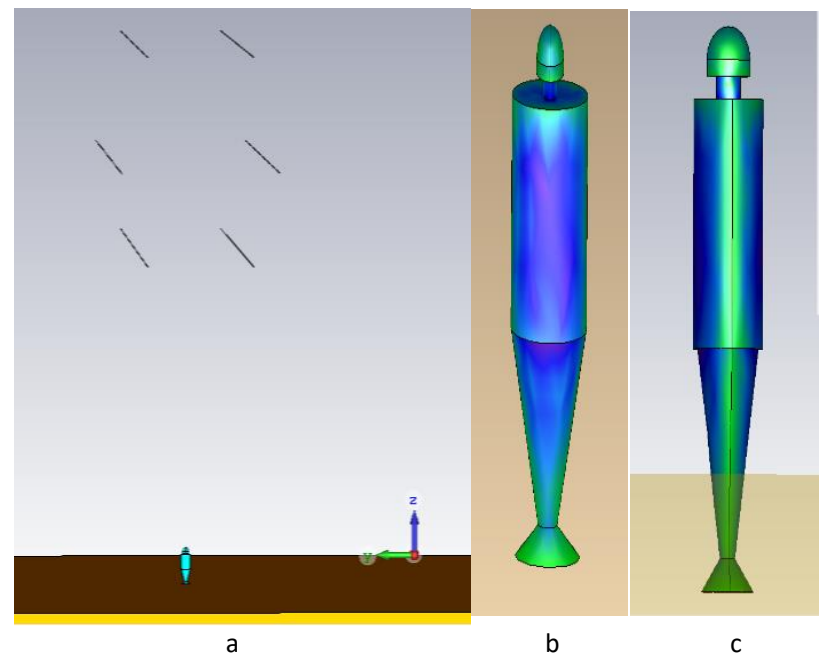

Figure 3. Working space of the simulation (a). The current density distribution on the model surface is very slightly influenced by the trunk relative orientation (b) parallel to the network plane and (c) normal to network. 


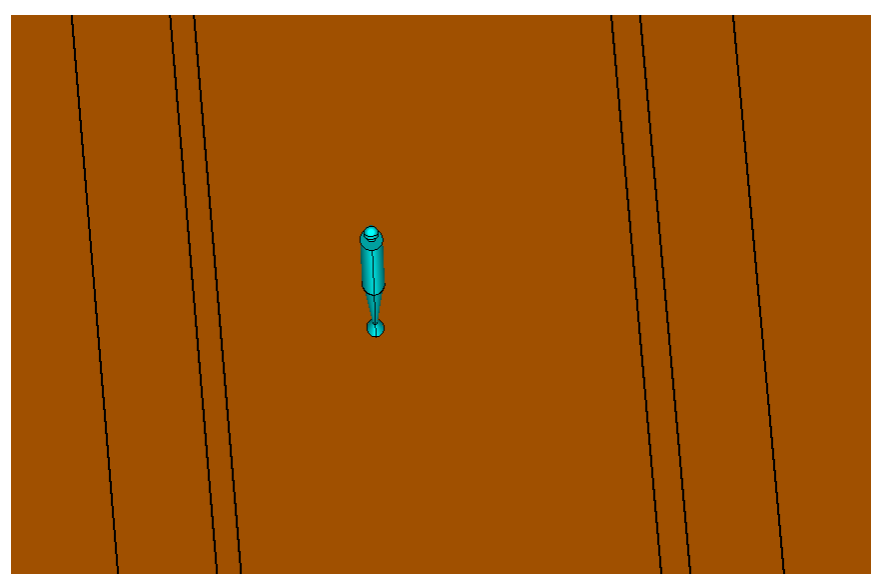

Figure 4. The trunk of the human body, parallel to the plane of the power network, in the most exposed position to inductive coupling.

the value of $2 \mathrm{~mA} / \mathrm{m}^{2}$ - the accepted admissibility threshold for residential exposure.

The corresponding area to be intersected by the magnetic field lines is the largest possible, as shown in Figure 4.

In Figure 5, we present the induced current density in the same horizontal elliptical cross-section performed $1 \mathrm{~m}$ high from the ground, this time with the great axis of the ellipse being normal to the network plane and, consequently, the coupling being minimal.

This situation is suggestively represented in Figure 6, the human trunk being rotated $90^{\circ}$ from the condition presented in the previous figure.

Up and down these two extremes are the intermediate values associated with the angles of $30^{\circ}, 45^{\circ}, 60^{\circ}$ between the large axis of the cross-section ellipse and the plane determined by the power network pillars. As expected, the value of the current density induced in the trunk decreases in proportion to the surface exposed to the field lines.

\section{CONCLUSIONS}

The modelling and simulations presented in this article are consistent with those obtained through the use of other software or communicated in significant journals, demonstrating the

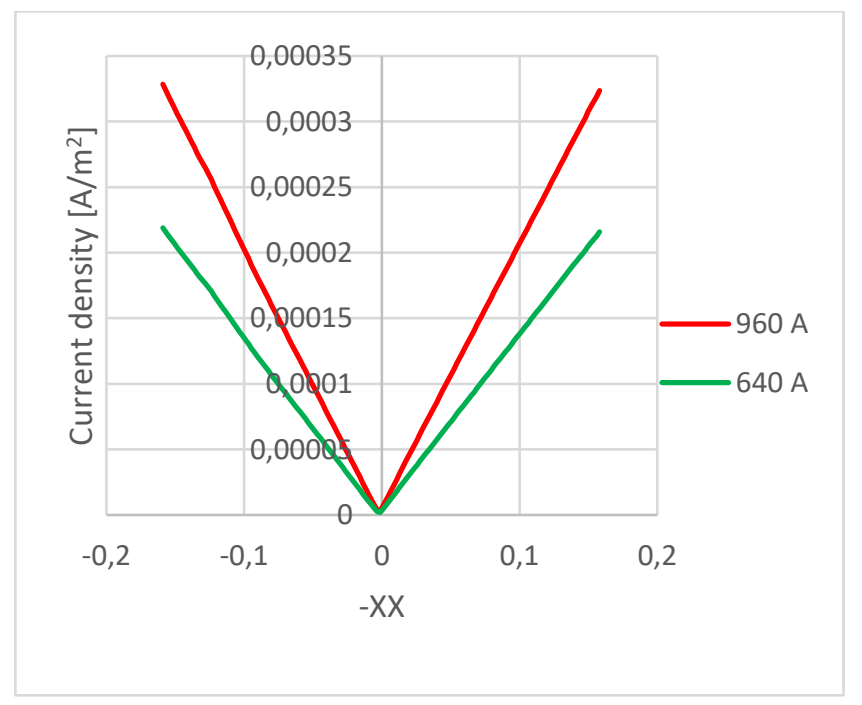

Figure 5. The trunk of the human body, parallel to the plane of the power network, in the most exposed position to inductive coupling

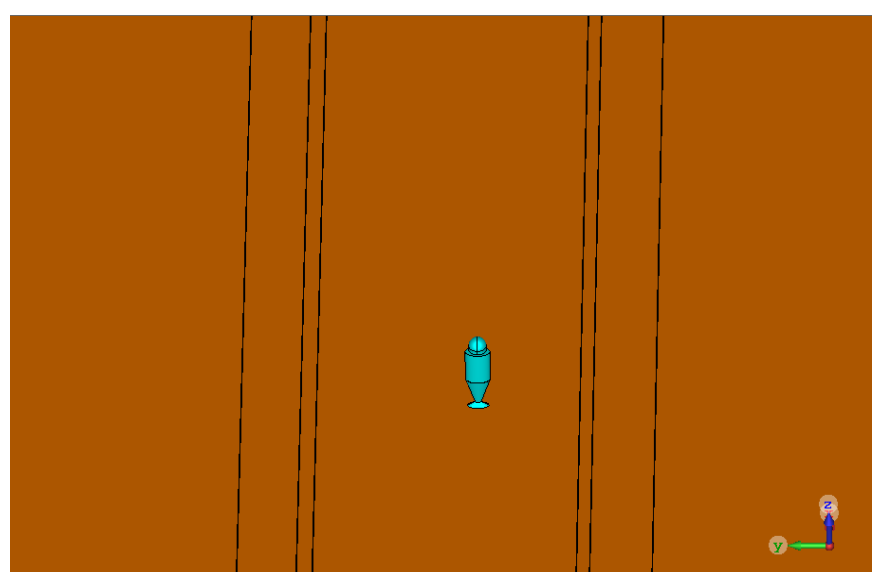

Figure 6. The trunk of the human body, normal to the plane of the power network, in the least exposed position to inductive coupling.

important resources of CST Studio Suite and the importance of the study of Extremely Low Frequency (ELF) electrical and magnetic fields, such as $50 \mathrm{~Hz}$. Because the human trunk has been modelled as an elliptic cylinder, it has been possible to study the distribution of the loop currents induced by the horizontal component of the magnetic field generated by overhead power transmission networks, both on the peripheral perimeter associated with the large axis of the cross-section and on that associated with the small one.

The graphic representations in Figure 3 and Figure 5 are in the form of a V - with almost straight arms. Interpreting these results, a good correspondence with the theory can be established, the induced current density being proportional to the radius of the exposed surface, under the theoretical condition of uniform electrical conductivity.

In addition, an increase in the RMS value of the current by $50 \%$ (from $640 \mathrm{~A}$ to $960 \mathrm{~A}$ ) resulted in an increase of approximately the same percentage of the current density induced (obviously, at the same point of the selected model).

The results herein obtained provide useful recommendations, especially for maintenance employees, who work close to highvoltage lines and should therefore avoid (as much as possible) the position with the large axis of the trunk being parallel to the plane of the supporting pillars.

Specifically, in the cases presented herein, there is a $57 \%$ reduction in exposure when the large axis of the elliptical cylinder that shapes the human trunk is oriented perpendicular to the active wires compared to the situation when it is oriented parallel to them - in other words, a reduction of the current density from 3.75 to $2.15 \mathrm{~mA} / \mathrm{m}^{2}$ for the case of the $640 \mathrm{~A}$ network, and a reduction from 5.5 to $3.2 \mathrm{~mA} / \mathrm{m}^{2}$ for the 960 A network.

According to the theoretical predictions, this reduction is approximately equal to the ratio between the small and the large radius of the ellipse representing the cross-section of the modelled trunk.

This reduction is all the more important as the involved values are within the sensitive range between occupational and residential basic restrictions. The recommendation becomes even more stringent for workers who are closer to the wires during the execution of urgent interventions.

In future work, we aim to perform a more refined study, which, based on successive $10^{\circ}$ rotations, will allow us to identify the position for which the total cumulative inductive coupling is minimal, simultaneously taking into account the induced currents on both the maximum and minimum perimeter. 
We will also consider that at a height of about $1 \mathrm{~m}$ from the ground, the human body has sensitive organs, such as the liver, spleen, and lungs, which have different conductivity and permittivity (and different basic restrictions). Taking these nonuniformities into account should lead to significant and even asymmetric nonlinearities in the two arms of the $\mathrm{V}$-shaped graphs presented in Figure 3 and Figure 5 herein. It is worth studying the possible overcoming of basic restrictions in certain organs considered more sensitive.

\section{REFERENCES}

[1] European Commission, Directive 2013/35/EU of the European Parliament and of the council of 26 June 2013 on the minimum health and safety requirements regarding the exposure of workers to the risks arising from physical agents (electromagnetic fields), Official J. Eur. Union, 2013, 1791, pp. 1-21J.

[2] C. M. Furse, O. P. Gandhi, Calculation of electric fields and currents induced in a millimeter-resolution human model at $60 \mathrm{~Hz}$ using the FDTD method, Bioelectromagnetics 19 (1998) pp. 293299. Online [Accessed 28 September 2020]

DOI: $\quad$ https://doi.org/10.1002/(SICI)1521 186X(1998)19:5\%3C293::AID-BEM3\%3E3.0.CO;2-X

[3] CST Studio Suite, Electromagnetic field simulation software, Dassault Systèmes,

URL: https://www.cst.com/

[4] Z. Liang, Y. Jiang, J. He, D. Jiang, F. Guo, Induced current in human body by electric field of overhead lines, Proc. of the $3^{\text {rd }}$ International Conference on Computer Science and Network Technology, Dalian, China,12-13 October 2013, pp. 101 - 104 DOI: https://doi.org/10.1109/ICCSNT.2013.6967072

[5] D. Medve, L. Mišenčík, M. Kolcun, J. Zbojovský, M. Pavlík Measuring of magnetic field around power lines, Proc. of the $8^{\text {th }}$ International Scientific Symposium Elektroenergetika, Stara Lesna, Slovakia, 16-18 September 2015, ISBN: 9781510811423 pp. $148-151$

[6] S. Ahmadi, A. A. Mohseniand, A. Shayegani Akmal, Electromagnetic fields near transmission lines - problems and solutions, Iranian Journal of Environmental Health, Science and Engineering 7(2) (2010) pp. 181-188. Online [Accessed 28 September 2020]

URL:

https://ijehse.tums.ac.ir/index.php/iehse/article/view/246/245

[7] D. Irimia, E. C. Bobric, Determination of induced currents in human body sitting under an overhead power line, Proc. of the $9^{\text {th }}$ International Symposium on Advanced Topics in Electrical Engineering (ATEE), Bucharest, Romania, 7-9 May 2015, pp. 404407.

DOI: https://doi.org/10.1109/ATEE.2015.7133837

[8] N. Korovkin, V. Goncharov, Calculation of induced EMF by overhead lines, Proc. of the IEEE NW Russia Young Researchers in Electrical and Electronic Engineering Conference (EIConRusNW), St. Petersburg, Russia, 2-3 February 2016, pp.608-610.

DOI: https://doi.org/10.1109/EIConRusNW.2016.7448256

[9] Hatem El Bidweihy, H. Anis, Comparative exposure to magnetic fields of live-line workers on power lines, PES T\&D, Orlando, FL,
USA, 7-10 May 2012, pp. 1-8.

DOI: https://doi.org/10.1109/TDC.2012.6281414

[10] X. Wu, D. J. Meisner, K. D. Stechschulte, V. Simha, R. J. Wellman, M. Thakur, K. R. Posey, Induced voltage \& current simulations, safety criterion, and mitigations for EHV transmission lines in close proximity, Proc. of the IEEE Industry Applications Society Annual Meeting (IAS), Portland, OR, USA, 23-27 September 2018, pp. 1-8.

DOI: https://doi.org/10.1109/IAS.2018.8544510

[11] B. Kuznetsov, I. Bovdui, A. Voloshko, T. Nikitina, Modeling and active shielding of magnetic field in residential buildings located near group of high voltage power lines, Proc. of the 2018 IEEE $3^{\text {rd }}$ International Conference on Intelligent Energy and Power Systems (IEPS), Kharkiv, Ukraine, 10-14 September 2018. DOI: https://doi.org/10.1109/IEPS.2018.8559488

[12] E. G. Ögel, S. Özen, S. Helhel, Evaluation of the electric and magnetic field levels of around the medium voltage power lines in related to public health, Proc. of the $15^{\text {th }}$ National Biomedical Engineering Meeting, Antalya, Turkey, 21-24 April 2010. DOI: https://doi.org/10.1109/BIYOMUT.2010.5479744

[13] D. Xudong, W. Guangning, B. Cao, J. Wu, Influence analysis of soil resistivity to induced voltage and current for transmission systems, Proc. of the IEEE Symposium on Electrical \& Electronics Engineering (EEESYM), Kuala Lumpur, Malaysia, 24-27 June 2012.

DOI: https://doi.org/10.1109/EEESym.2012.6258710

[14] M. V. Paulet, C. Lazarescu, A. Salceanu, Modeling the currents induced in the human body by an overhead high voltage power line, Proc. of the 10 $10^{\text {th }}$ International Conference and Exposition on Electrical and Power Engineering (EPE 2018), Iasi, Romania, 18 19 October 2018. DOI: https://doi.org/10.1109/ICEPE.2018.8559928

[15] M. V. Paulet, C. Lazarescu, O. Bejenaru, A. Salceanu, Study on induced currents in an elliptical cylindrical model by overhead high voltage power lines, Proc. of the 11 th International Symposium Advanced Topics in Electrical Engineering (ATEE), Bucharest, Romania, 28-30 March 2019. DOI: https://doi.org/10.1109/ATEE.2019.8724870

[16] M. V. Paulet, A. Salceanu, C. Lazarescu, O. Bejenaru, B. D. Alistar, Study upon the influence of human body torso stance on the inductive coupling, Proc. of the $23^{\text {rd }}$ IMEKO TC 4 International Symposium, Xi'an, China, 17-20 September 2019, pp. 181-185, ISBN 978-606-13-5238-8. Online [Accessed 29 September 2020]

URL: https://www.imeko.org/publications/tc4-2019/IMEKOTC4-2019-039.pdf

[17] A. Salceanu, M. Paulet, B. D. Alistar, O. Asiminicesei, Upon the contribution of image currents on the magnetic fields generated by overhead power lines, Proc. of the $12^{\text {th }}$ International Conference on Electromechanical and Power Systems (SIELMEN), 10-11 Oct., 2019, Chisinau, Rep. of Moldova, pp 199-204, ISBN: 978-1-7281-4011-7. DOI: https://doi.org/10.1109/SIELMEN.2019.8905880

[18] A. Salceanu, E. Lunca, B. D. Alistar, S. Ursache, Upon the influence of charge image on the electric field intensity, Proc. of the $12^{\text {th }}$ International Conference on Electromechanical and Power Systems (SIELMEN), 10-11 October 2019, Chisinau, Rep. of Moldova, pp. 213-218, ISBN: 978-1-7281-4011-7. DOI: https://doi.org/10.1109/SIELMEN.2019.8905895 